\title{
Avaliação do conhecimento sobre a utilização de inaladores entre médicos e profissionais de farmácia dos Açores
}

Sofia Correia, ${ }^{1}$ Fábio Luz, ${ }^{2}$ Vanessa Amaral, ${ }^{3}$ Adelino Dias, ${ }^{4}$ Telma Miragaia ${ }^{5}$

\section{RESUMO}

Objetivos: Avaliar a demonstração prática e a descrição oral da utilização do inalador pressurizado e inalador em pó seco de médicos e profissionais de farmácia.

Tipo de estudo: Observacional, multicêntrico, analítico, transversal.

Local: Centros de saúde, serviços de pediatria e medicina interna e farmácias de quatro ilhas dos Açores.

População: Médicos e profissionais de farmácia.

Métodos: Nos médicos foi efetuado um censo da população, enquanto nos profissionais de farmácia foi feita uma amostra de conveniência. Os profissionais simularam o uso de dois dispositivos inalatórios e os autores verificaram o cumprimento de cada etapa numa checklist. Para a análise dos dados foi utilizada a regressão logística binária.

Resultados: Participaram no estudo 45 profissionais de farmácia, 91 médicos de família, 26 internistas e 19 pediatras com idade média de 40,8 anos ( $\pm 12,2), 66,9 \%$ (121) eram mulheres. Cinco (2,8\%) demonstraram corretamente a técnica inalatória de ambos os dispositivos. As etapas em que mais erraram foram: a expiração máxima antes da inalação, suster a respiração pelo menos cinco segundos em ambos os inaladores, lavar a boca com água e agitar o inalador pressurizado antes de usar. No inalador pressurizado, um conhecimento pobre da técnica inalatória está relacionado com o facto de ser profissional de farmácia $(O R=4,94)$, idade entre 51 e 65 anos $(O R=5,95)$ e explicar "às vezes, nem sempre ou nunca" a técnica $(O R=3,63)$. Relativamente ao inalador pó seco observou-se que ter um conhecimento pobre da técnica inalatória está associado com o facto de ser profissional de farmácia $(O R=3,51)$, internista $(O R=6,55)$, idade entre 36 e 50 anos $(O R=3,68), 51$ e 65 anos $(O R=11,44)$ e exercer na ilha do Faial $(O R=15,98)$.

Conclusões: A maioria dos profissionais de saúde falha na explicação de várias etapas essenciais para o uso correto dos dispositivos inalatórios.

Palavras-chave: Dispositivos Inalatórios; Técnica Inalatória; Arquipélago dos Açores.

\section{INTRODUÇÃO}

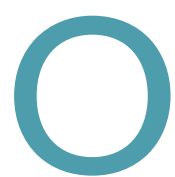

s inaladores são um componente fundamental no tratamento das doenças das vias respiratórias mais prevalentes, como a asma ou a doença pulmonar obstrutiva crónica, porque permitem o transporte e obtenção de

\footnotetext{
1,2,3,5 Médicos internos de Medicina Geral e Familiar

${ }^{4}$ Assistente Graduado Sénior de Medicina Geral e Familiar

1,4,5 Unidade de Saúde de Itha de São Miguel

¿Unidade de Saúde da llha Terceira

${ }^{3}$ Unidade de Saúde da llha do Pico
}

concentrações elevadas dos fármacos nas vias respiratórias inferiores com doses menores, obtendo-se um início de ação mais rápido, maximizando os efeitos terapêuticos e minimizando a absorção sistémica e os possíveis efeitos secundários correspondentes. ${ }^{1-4}$

Se incorretamente utilizados, os fármacos não alcançam as vias respiratórias inferiores em quantidade suficiente, não atingindo concentrações terapêuticas eficazes, pelo que uma boa técnica inalatória é fundamental para o controlo destas patologias. ${ }^{1}$ Está provado que a efetividade do tratamento com um inalador 
depende da técnica utilizada. ${ }^{4-5}$ Giraud $^{6}$ verificou que o uso indevido de inaladores estava associado à diminuição de controlo da asma e Finkn ${ }^{7}$ afirma que a gestão bem sucedida da asma é $10 \%$ medicamento e $90 \%$ de educação. Os profissionais de saúde que lidam com doentes com patologias nas quais é imperativo o uso de inaladores, como médicos e profissionais de farmácia, são responsáveis pelo ensino da técnica de utilização destes aparelhos, desempenhando assim um papel crucial no alcance dos objetivos terapêuticos. ${ }^{1,-9} \mathrm{Na}$ revisão de Lavorini et al $^{5}$ verificou-se que entre 4 a $94 \%$ dos pacientes não usavam corretamente os seus inaladores e 25\% deles nunca tinham recebido instruções verbais sobre a técnica inalatória. Há evidência de que o ensino da técnica inalatória por um profissional de saúde está associado a uma maior probabilidade dos doentes usarem os dispositivos corretamente. ${ }^{9}$

Os inaladores pressurizados e inaladores de pó seco são dispositivos frequentemente recomendados..$^{10} \mathrm{Em}$ Portugal Continental existem poucos estudos publicados que avaliam os conhecimentos dos profissionais de saúde sobre a técnica de utilização de inaladores pressurizados e inaladores de pó seco. No estudo de Silveira, ${ }^{11}$ que englobou internistas, o autor concluiu que os dispositivos inalatórios eram incorretamente manuseados pelo grupo testado, apesar de frequentemente prescritos. A nível internacional, desde a década de 80 que têm sido realizados trabalhos para avaliar o conhecimento e habilitação prática do uso de inaladores entre os profissionais de saúde, mas os resultados continuam aquém do esperado. ${ }^{12-16}$ Nos Açores não existe nenhum estudo publicado desta natureza. Assim, este trabalho teve como objetivo avaliar a demonstração prática e a descrição oral da utilização do inalador pressurizado e inalador de pó seco de médicos dos cuidados de saúde primários, dos serviços de medicina interna, serviços de pediatria e profissionais de farmácia das ilhas de São Miguel, Terceira, Pico e Faial.

\section{MÉTODOS}

Realizou-se um estudo observacional transversal e analítico, com recolha de dados entre 1 de novembro e 14 de dezembro de 2013. Tratou-se dum estudo multicêntrico realizado em quatro ilhas do arquipélago dos Açores (São Miguel, Terceira, Faial e Pico).

A população em estudo correspondeu aos médicos do Hospital do Divino Espírito Santo na ilha de São Miguel e Santo Espírito na Ilha Terceira (serviços de medicina interna e pediatria), das Unidades de Saúde de Ilha de São Miguel, Terceira, Faial e Pico e profissionais de farmácias comunitárias das mesmas ilhas. Pretendeu-se fazer um censo de todos os médicos dos referidos locais e a nível das farmácias foi utilizada uma amostra de conveniência composta por um profissional por farmácia, uma vez que não era possível isolar estes profissionais sem que os outros colegas observassem a demonstração. De acordo com a listagem disponibilizada pelos recursos humanos dos hospitais e unidades de saúde de ilha, exerciam funções nas referidas intuições 198 médicos. O número de farmácias foi obtido por consulta do site www.farmaciasportuguesas.pt. Foram excluídos 24 médicos: 12 por terem participado no estudo piloto e os restantes por terem acesso ao protocolo de investigação que continha a checklist com a descrição das etapas da técnica de cada inalador.

As variáveis estudadas foram: idade, género, profissão, local de trabalho, anos de serviço, disponibilidade para explicação sobre o funcionamento dos inaladores e opinião sobre que profissionais devem explicar o modo de funcionamento (variáveis independentes). A variável dependente estudada foi o conhecimento sobre a técnica de utilização de inaladores (pressurizado e pó seco - turbohaler), sendo que foi considerado um pobre conhecimento quando o participante falhava quatro ou mais etapas.

Para determinar o conhecimento sobre a utilização de inaladores foi elaborada uma checklist composta por sete etapas para o inalador pressurizado e seis etapas para o turbohaler (Anexo I), tendo sido adaptadas das recomendações nacionais do Grupo de Doenças Respiratórias da Associação Portuguesa de Medicina Geral e Familiar. ${ }^{17}$

A recolha de dados foi efetuada mediante um inquérito elaborado e ministrado pelos autores. $\mathrm{O}$ inquérito era composto por um cabeçalho numerado contendo as variáveis demográficas e em estudo. $\mathrm{O}$ inquérito foi objeto de um estudo piloto aplicado a 12 pessoas do universo em estudo que permitiu avaliar os vários aspetos metodológicos e aperfeiçoar a técnica de recolha de dados. Para minimizar o viés do entrevistador foi elaborado um texto padrão para os quatro in- 
vestigadores utilizarem. Os investigadores entregaram aos participantes o consentimento informado que foi assinado por ambos. Foram entregues sequencialmente os dois inaladores e o participante explicava a técnica de utilização de cada um deles como se estivesse a ensinar o doente, enquanto o investigador assinalava na checklist as etapas cumpridas. Foram pontuadas como corretas as etapas demonstradas pelo participante, assim como a sua descrição oral, visto que o objetivo dos investigadores era aproximar o ambiente de demonstração da prática clínica diária do profissional, pois nem sempre existe um dispositivo placebo para demonstração. Considerou-se que o participante tinha um pobre conhecimento sobre a técnica inalatória quando falhava quatro ou mais etapas e um adequado conhecimento quando falhava entre zero e três etapas.

Para a análise dos dados obtidos, estes foram codificados e posteriormente informatizados utilizando o programa SPSS, versão 20.0. A abordagem inicial passou por análise descritiva e posteriormente foi efetuada uma análise de regressão logística bivariável para analisar as associações individuais com a variável dependente (falhar quatro ou mais etapas). As variáveis que se mostraram associadas na análise univariável e sem colinearidade espectável foram incluídas no modelo de regressão logística multivariável. O nível de significância adotado foi de 0,05 .

O projeto de investigação teve parecer favorável da Comissão de Ética do Hospital do Divino Espírito Santo na ilha de São Miguel e Santo Espírito na Ilha Terceira.

\section{RESULTADOS}

Participaram no estudo 136 médicos de um total de 174, o que corresponde a uma taxa de participação de $78,2 \%$, e 45 profissionais de farmácia de um total de 58 farmácias, obtendo-se uma taxa de participação de $77,6 \%$ entre os últimos.

Dos 181 profissionais que integraram o trabalho de investigação, 66,9\% (121) eram mulheres. A idade dos inquiridos variou entre 24 e 65 anos, com uma média de $40,8( \pm 12,2)$ anos (Quadro I).

Cinco $(2,8 \%)$ demonstraram corretamente a técnica inalatória de ambos os dispositivos. Em média, os participantes falharam $3,1( \pm 1,5)$ etapas no inalador pressurizado e 2,5 $( \pm 1,4)$ etapas no inalador de pó seco. As etapas em que os profissionais mais erraram em ambos

\begin{tabular}{|c|c|c|}
\hline & $\mathbf{n}$ & $\%$ \\
\hline \multicolumn{3}{|l|}{ Género } \\
\hline Feminino & 121 & 66,9 \\
\hline Masculino & 60 & 33,1 \\
\hline \multicolumn{3}{|l|}{ Profissão } \\
\hline Médico de MGF & 91 & 50,3 \\
\hline Internista & 26 & 14,4 \\
\hline Pediatra & 19 & 10,5 \\
\hline Profissional de farmácia & 45 & 24,9 \\
\hline \multicolumn{3}{|l|}{ Itha } \\
\hline São Miguel & 105 & 58,0 \\
\hline Terceira & 49 & 27,1 \\
\hline Pico & 17 & 9,4 \\
\hline Faial & 10 & 5,5 \\
\hline
\end{tabular}

Nota: MGF- Medicina Geral e Familiar.

os inaladores foram: a expiração máxima antes da inalação, o suster a respiração pelo menos cinco segundos e a lavagem da boca com água se o inalador contivesse corticoides (Quadro II). No inalador pressurizado, a etapa 1 (agitar o inalador pressurizado antes de usar) foi também com frequência omitida por 60,2\% (109) participantes.

Relativamente ao inalador pressurizado, dos 181 participantes 39,8\% (72) falharam quatro ou mais etapas. Ao realizar o modelo de regressão logística simples (Quadro III) encontrou-se diferença estatisticamente significativa entre falhar quatro ou mais etapas (conhecimento pobre da técnica) e ser profissional de farmácia $(\mathrm{p}=0,005)$, faixa etária [36, 50] (p=0,032) e [51-65] $(p=0,001)$. Foi encontrada uma associação entre conhecimento adequado da técnica (falhar menos de quatro etapas) e ser pediatra ( $\mathrm{p}=0,034)$ e explicar "sempre ou quase sempre" a técnica inalatória $(p=0,001)$. Ao realizar análise multivariável foram excluídas do modelo as variáveis anos de serviço, investigador e ilha, visto que não acrescentavam valor explicativo ao mesmo. O Quadro IV mostra os resultados após ajustamento para profissão, idade e frequência de explicação da técnica. As classes de referência para as variáveis independentes foram médico de família, faixa etária $[20,35]$ 
QUADRO II. Proporção de participantes que falharam cada etapa

Inalador pressurizado

1. Agitar o inalador doseável antes de usar.

2. Segurar o aerossol com a base da cápsula de metal voltada para cima, entre os dedos com o polegar sobre a base.

3. Esvaziar os pulmões tanto quanto possível (expiração máxima).

4. Ajustar a boca firmemente em torno do bucal.

5. Inspirar pela boca progressivamente e comprimir o inalador.

6. Suster a respiração pelo menos 5 segundos.

7. Lavar a boca com água se o inalador contiver corticoides.

Inalador de pó seco

1. Rodar a base nos dois sentidos até ouvir um click.

2. Esvaziar os pulmões tanto quanto possível (expiração máxima).

3. Ajustar a boca firmemente em torno do bucal.

4. Inspirar pela boca rápida e progressiva.

5. Suster a respiração pelo menos 5 segundos.

6. Lavar a boca com água se o inalador contiver corticoides.

\begin{tabular}{|r|r|r|}
$\mathbf{n}$ & \multicolumn{1}{|c|}{$\%$} & IC $95 \%$ \\
109 & 60,2 & $53,0-67,4$ \\
42 & 23,2 & $17,0-29,4$ \\
& & \\
87 & 48,1 & $40,7-55,4$ \\
8 & 4,4 & $1,4-7,4$ \\
62 & 34,3 & $27,3-41,2$ \\
108 & 59,7 & $52,5-66,9$ \\
157 & 86,7 & $81,8-91,7$ \\
& & \\
54 & 29,8 & $23,1-36,6$ \\
92 & 50,8 & $43,5-58,2$ \\
13 & 7,2 & $3,4-11,0$ \\
26 & 14,4 & $9,2-19,5$ \\
115 & 63,5 & $56,5-70,6$ \\
149 & 82,3 & $76,7-87,9$
\end{tabular}

e frequência de explicação "sempre ou quase sempre". $O$ valor de adequação do modelo foi $\mathrm{R}^{2}=0,23$. Os resultados mostram que a probabilidade de ter um conhecimento pobre da técnica inalatória está relacionada com ser profissional de farmácia $(\mathrm{OR}=4,94)$, ter idade entre 51 e 65 anos $(\mathrm{OR}=5,95)$ e explicar "às vezes, nem sempre ou nunca" a técnica ( $\mathrm{OR}=3,63)$.

Relativamente ao inalador pó seco, dos 181 participantes $20,4 \%$ (37) falharam quatro ou mais etapas. Ao realizar o modelo de regressão logística simples (Quadro III) encontrou-se diferença estatisticamente significativa entre falhar quatro ou mais etapas (conhecimento pobre da técnica) e faixa etária [36, 50] $(\mathrm{p}=0,026)$, [51-65] $(p=0,001)$ e ilha do Faial $(p=0,007)$. Ao realizar análise multivariável foram excluídas do modelo as variáveis anos de serviço, investigador e frequência de explicação, visto que não acrescentavam valor explicativo ao mesmo. O Quadro V mostra os resultados após ajustamento para profissão, idade e ilha. As classes de referência para as variáveis independentes foram médico de família, faixa etária [20,35] e ilha de São Miguel. $\mathrm{O}$ valor de adequação do modelo foi $\mathrm{R}^{2}=0,21$. Os resultados mostram que a probabilidade de ter um conhe- cimento pobre da técnica inalatória está relacionado com ser profissional de farmácia $(\mathrm{OR}=3,51)$, internista $(\mathrm{OR}=6,55)$, ter idade entre 36 e 50 anos $(\mathrm{OR}=3,68), 51 \mathrm{e}$ 65 anos $(\mathrm{OR}=11,44)$ e ilha do Faial $(\mathrm{OR}=15,98)$.

Um dos investigadores utilizou um inalador pressurizado de cor lilás contendo uma associação entre corticoide e agonista beta 2 de longa-ação, enquanto os restantes investigadores utilizaram um dispositivo de cor azul contendo salbutamol. Quanto ao inalador de pó seco, um dos investigadores utilizou um dispositivo com base azul contendo terbutalina e os restantes usaram um dispositivo com base vermelha contendo uma associação entre corticoide e agonista beta 2 de longaação. Ao aplicar a regressão logística simples, não se observou relação entre a etapa "Lavar a boca com água se o inalador contiver corticoides" e a cor do inalador utilizado, tanto pressurizado $(\mathrm{p}=0,84)$ como de pó seco $(\mathrm{p}=0,12)$.

De todos os participantes, 77,3\% (140) afirmaram explicar sempre ou quase sempre o funcionamento dos dispositivos quando os prescreviam ou forneciam.

Dos profissionais de saúde que participaram no estudo, $59,7 \%$ (108) considera que médicos, farmacêuti- 
cos e enfermeiros são os profissionais responsáveis por explicar o funcionamento dos inaladores aos utentes. Aproximadamente $18,8 \%$ (34) acha que esta tarefa cabe aos médicos e farmacêuticos; $11 \%$ (20) aos médicos apenas; $7,7 \%$ (14) aos médicos e enfermeiros; $1,7 \%$ (3) aos farmacêuticos; 0,6\% (1) aos farmacêuticos e enfermeiros; e 0,6\% (1) aos médicos, farmacêuticos, enfermeiros e cuidadores.

\section{DISCUSSÃO}

Constatou-se que a maioria dos profissionais de saúde falha na explicação de várias etapas essenciais para o uso correto dos dispositivos inalatórios, o que está de acordo com os resultados de artigos nacionais e internacionais. ${ }^{11-16}$

Os profissionais de farmácia foram os participantes que revelaram um conhecimento mais pobre sobre ambos os inaladores. Isto poderá dever-se à falta de formação desses profissionais nesta área e ao facto de este ser um grupo heterogéneo que inclui, entre outros, profissionais que não frequentaram o ensino superior.

À medida que a idade dos profissionais aumenta, observa-se um conhecimento mais pobre relativamente à técnica inalatória. Isto pode ser justificado pela falta de formação específica nesta área como a participação em workshops e oficinas de técnica inalatória. Esta variável não fez parte da nossa investigação mas poderia revelar-se útil, pelo que estudos futuros que a englobem poderiam acrescentar mais informação sobre a sua influência no conhecimento da técnica inalatória.

A observação de que uma elevada percentagem de profissionais afirma explicar sempre ou quase sempre o funcionamento do inalador aos utentes, associada a um menor número de etapas falhadas, constituiu um aspeto positivo. Verificou-se, no entanto, que uma percentagem significativa dos que dizem explicar sempre ou quase sempre o funcionamento da técnica de ambos os inaladores falha 
quatro ou mais etapas (32,9\% no inalador pressurizado e $18,6 \%$ no inalador de pó seco).

Algumas limitações deste estudo que poderão ter contribuído para o enviesamento dos resultados foram: a dificuldade na avaliação objetiva das etapas “inspiração progressiva no inalador pressurizado" e "inalação vigorosa no inalador de pó seco"; algumas etapas da técnica inalatória não terem sido incluídas na checklist utilizada, como a remoção da tampa, verificação do número de doses ou cuidados a ter relativamente à limpeza do dispositivo; alguns profissionais poderem ter tido conhecimento prévio do objetivo do estudo através de outros colegas que já tinham participado; não ter sido feita a distinção entre farmacêuticos e técnicos de farmácia porque os autores consideraram que ambos são responsáveis pelo ensino da técnica inalatória independentemente do grau académico, tendo em conta que ambos os profissionais atendem os utentes na farmácia. $\mathrm{O}$ facto de ter sido efetuada uma amostra de conveniência nas farmácias poderá também constituir um viés de amostragem.

Concluiu-se com este trabalho de investigação que uma percentagem significativa de profissionais de saúde tem um conhecimento pobre sobre a técnica inalatória, falhando quatro ou mais
QUADRO IV. Análise multivariável segundo o modelo de regressão logística para a demonstração prática e a descrição oral da utilização do inalador pressurizado

\begin{tabular}{|c|c|c|c|c|}
\hline Variável & OR (IC 95\%) & $\mathbf{P}$ & OR ajustado (IC95\%)* & $\mathbf{P}$ \\
\hline \multicolumn{5}{|l|}{ rofissão } \\
\hline MGF & 1 & & & \\
\hline Farmácia & $2,90(1,39-6,07)$ & 0,005 & $4,94(1,96-12,42)$ & 0,001 \\
\hline Medicina interna & $1,94(0,80-4,68)$ & 0,14 & $2,42(0,87-6,76)$ & 0,09 \\
\hline Pediatria & $0,11(0,01-0,84)$ & 0,03 & $0,21(0,03-1,76)$ & 0,15 \\
\hline \multicolumn{5}{|l|}{ Eaixa etária } \\
\hline $20-35$ & 1 & & & \\
\hline $36-50$ & $2,26(1,07-4,76)$ & 0,03 & $1,66(0,69-3,98)$ & 0,26 \\
\hline $51-65$ & $3,70(1,75-7,80)$ & 0,001 & $5,95(2,46-14,44)$ & $<0,001$ \\
\hline \multicolumn{5}{|l|}{ Erequência de explicação } \\
\hline Sempre ou quase sempre & 1 & & & \\
\hline Às vezes, nem sempre, nunca & $3,54(1,71-7,33)$ & 0,001 & $3,63(1,57-8,38)$ & 0,003 \\
\hline
\end{tabular}

Modelo ajustado por profissão, faixa etária e frequência de explicação (Cox E Snell $R$ Square=0,23, Nagelkerke $R$ quare $=0,31)$

Nota: OR: Odds ratio. OR (IC 95\%): intervalo de confiança a 95\% para o odds ratio.

Nota: MGF-Medicina Geral e Familiar.

\begin{tabular}{|c|c|c|c|c|}
\hline Variável & OR (IC95\%) & $\mathbf{P}$ & OR ajustado (IC95\%)* & $\mathbf{P}$ \\
\hline \multicolumn{5}{|l|}{ Profissão } \\
\hline MGF & 1 & & & \\
\hline Farmácia & $1,90(0,82-4,41)$ & 0,13 & $3,51(1,10-11,23)$ & 0,04 \\
\hline Medicina interna & $2,08(0,77-5,62)$ & 0,15 & $6,55(1,76-24,40)$ & 0,005 \\
\hline Pediatria & 0,00 & 0,10 & 0,00 & 0,10 \\
\hline \multicolumn{5}{|l|}{ Faixa etária } \\
\hline 20-35 & 1 & & & \\
\hline $36-50$ & $3,04(1,14-8,08)$ & 0,03 & $3,68(1,15-11,77)$ & 0,03 \\
\hline $51-65$ & $4,98(1,95,12,71)$ & 0,001 & $11,44(3,26-40-14)$ & $<0,001$ \\
\hline \multicolumn{5}{|l|}{ Ilha } \\
\hline São Miguel & 1 & & & \\
\hline Terceira & $0,59(0,22-1,59)$ & 0,30 & $0,50(0,17-1,46)$ & 0,20 \\
\hline Faial & $6,38(1,64-24,73)$ & 0,007 & $15,98(2,99-85,41)$ & 0,001 \\
\hline Pico & $1,77(0,56-5,60)$ & 0,33 & $1,33(0,38-4,66)$ & 0,65 \\
\hline
\end{tabular}

* Modelo ajustado por profissão, faixa etária e ilha (Cox E Snell $R$ Square $=0,21$; Nagelkerke $R$ Square $=0,33$ ) Nota: OR: Odds ratio. OR (IC 95\%): intervalo de confiança a $95 \%$ para o odds ratio.

Nota: MGF-Medicina Geral e Familiar. 
etapas imprescindíveis para que os fármacos atinjam as vias respiratórias inferiores em concentrações terapêuticas. ${ }^{18}$ Há evidência de que o ensino da técnica inalatória por um profissional de saúde está associado a uma maior probabilidade dos doentes usarem os dispositivos corretamente, pelo que programas de ensino devem ser implementados para melhorar o desempenho dos profissionais (médicos, enfermeiros, fisioterapeutas, técnicos de farmácia) e, consequentemente, dos utentes. ${ }^{9,19-20}$ Programas desta natureza existem em Portugal sob a forma de workshop/oficina com cerca de 20 participantes em que são manuseados todos os tipos de dispositivos inalatórios existentes no mercado, mas ainda não estão ao alcance de todos os profissionais de saúde. Neste estudo foi dado um feedback aos participantes através do envio por email da checklist utilizada e de folhetos informativos sobre os passos da técnica inalatória.

\section{AGRADECIMENTOS}

Aos Orientadores de Formação dos autores: Dra. Ana Marques, Dr. Sampaio Duarte, Dra. Lucília Mendes e Dra. Carmina Pais. Ao Dr. Daniel Pinto pela ajuda na revisão do artigo.

\section{REFERÊNCIAS BIBLIOGRÁFICAS}

1. Fink JB. Metered-dose inhalers, dry powder inhalers, and transitions. Respir Care. 2000;45(6):623-35.

2. Torgal J, editor.Vigilância global, prevenção e controlo das doenças respiratórias crónicas: uma abordagem integradora. Lisboa: Direção-Geral da Saúde; 2007. ISBN 9789726751830

3. Global Initiative for Asthma. Global strategy for asthma management and prevention for adults and children older than 5 years. Global Initiative for Asthma; 2010 [updated 2012; cited 2013 Jul 12]. Available from: http://www.ginasthma.org/local/uploads/files/GINA_Report_March13.pdf

4. Dolovich MB, Ahrens RC, Hess DR, Anderson P, Dhand R, Rau JL, et al. Device selection and outcomes of aerosol therapy: evidence-based guidelines: American College of Chest Physicians/American College of Asthma, Allergy, and Immunology. Chest. 2005;127(1):335-71.

5. Lavorini F, Magnan A, Dubus JC, Voshaar T, Corbetta L, Broeders M, et al. Effect of incorrect use of dry powder inhalers on management of patients with asthma and COPD. Respir Med. 2008;102(4):593-604.

6. Giraud V, Roche N. Misuse of corticosteroid metered-dose inhaler is associated with decreased asthma stability. Eur Respir J. 2002;19(2): 246-51.

7. Fink JB. Inhalers in asthma management: is demonstration the key to compliance? Respir Care. 2005;50(5):598-600.

8. Self TH, Brooks JB, Lieberman P, Ryan MR. The value of demonstration and role of the pharmacist in teaching the correct use of pressurized bronchodilators. Can Med Assoc J. 1983;128(2):129-31.

9. Hesselink AE, Penninx BW, van der Windt DA, van Duin BJ, de Vries P, Twisk JW, et al. Effectiveness of an education programme by a general practice assistant for asthma and COPD patients: results from a randomised controlled trial. Patient Educ Couns. 2004;55(1):121-8.

10. Direção-Geral da Saúde. Normas para a abordagem e controlo da asma: norma $n^{\circ}$ 16/2011, de 27/09/2011, atualizada a 14/06/2012). Lisboa: DGS; 2012.

11. Silveira P, Rocha L, Ferreira J.Técnicas de medicação inalatória: saberão os clínicos utilizar os dispositivos inalatórios? Rev Port Imunoalergol. 1998;5(1):37-42.

12. Guidry GG, Brown WD, Stogner SW, Georgé RB. Incorrect use of metered dose inhalers by medical personnel. Chest. 1992;101(1):31-3.

13. Interiano B, Guntupalli KK. Metered-dose inhalers: do health care providers know what to teach? Arch Intern Med. 1993;153(1):81-5.

14. Kelling JS, Strohl KP, Smith RL, Altose MD. Physician knowledge in the use of canister nebulizers. Chest. 1983;83(4):612-4.

15. Plaza V, Sanchis J, Roura P, Molina J, Calle M, Quirce S, et al. Physicians' knowledge of inhaler devices and inhalation techniques remains poor in Spain. J Aerosol Med Pulm Drug Deliv. 2012;25(1):16-22.

16. Hanania NA, Wittman R, Kesten S, Chapman KR. Medical personnel's knowledge of and ability to use inhaling devices: metered-dose inhalers, spacing chambers, and breath-actuated dry powder inhalers. Chest. 1994;105(1):111-6.

17. Silva E, Pina A, Quelhas A, Cruz AM. Passos da técnica inalatória [Internet]. Lisboa:Associação Portuguesa de Medicina Geral e Familiar; 2012. Available from: http://www.apmgf.pt/ficheiros/Folheto_tecnica_inalatoria_GRESP.pdf

18. Rootmensen GN, Van Keimpema AR, Jansen HM, de Haan RJ. Predictors of incorrect inhalation technique in patients with asthma or COPD: a study using a validated videotaped scoring method. J Aerosol Med Pulm Drug Deliv. 2010;23(5):323-8.

19. Al-Jahdali H, Ahmed A, Al-Harbi A, Khan M, Baharoon S, Bin Salih S, et al. Improper inhaler technique is associated with poor asthma control and frequent emergency department visits. Allergy Asthma Clin Immunol. 2013;9(1):8.

20. Yildiz F, Asthma Inhaler Treatment Study Group. Importance of inhaler device use status in the control of asthma in adults: the asthma inhaler treatment study. Respir Care. 201459(2):223-30.

\section{CONFLITO DE INTERESSES}

Os autores declaram não ter conflitos de interesses.

\section{ENDEREÇO PARA CORRESPONDÊNCIA}

Sofia Feijó Correia

Rua Natividade, nº 9, 9560-215 Lagoa

E-mail: sofia.fj.correia@gmail.com

Recebido em 12-05-2014

Aceite para publicação em 19-01-2015 
ABSTRACT

\section{PHYSICIANS' AND PHARMACISTS' KNOWLEDGE OF INHALER TECHNIQUE IN THE AZORES}

Objectives: To evaluate physicians' and pharmacists' knowledge and competence in the use of pressurized metered dosed inhalers and turbohalers.

Study Design: Multicenter cross-sectional.

Setting: Primary healthcare centers, pediatric and internal medicine services, and pharmacies on four Azorean islands.

Participants: Family physicians, internists, pediatricians, and pharmacists.

Methods: A census of the population of physicians and a convenience sample of pharmacists were obtained. Health care providers were asked to demonstrate the use of two inhaler devices. The authors observed each step using a checklist. Logistic regression was used for data analysis.

Results: The study population included 45 pharmacists, 91 family doctors, 26 internists and 19 pediatricians. The mean age of participants 40.8 years $( \pm 12.2)$, and $66.9 \%(121)$ of the sample were women. Five participants $(2.8 \%)$ correctly demonstrated inhalation technique with both devices. The most prevalent errors observed were failing to exhale to residual volume, failing to hold the inhaled breath for 5 seconds with both inhalation devices, failure to wash the mouth with water if the device contained steroids, and failure to shake the metered dosed inhaler before using it. Poor knowledge about inhalation technique with the metered dosed inhaler was related to being a pharmacist $(O R=4.94)$, age between 61 and 65 years $(O R=5.95)$, and explaining the technique to patients 'sometimes, almost never, or never' (OR=3.63). With the dry powder inhaler, poor knowledge about inhalation technique was related to being a pharmacist $(\mathrm{OR}=3.51)$, being an internist $(\mathrm{OR}=6.55)$, age between 36 and 50 years $(O R=3.68)$, age between 51 and 65 years $(O R=11.44)$, and practicing in Faial island $(O R=15.98)$.

Conclusions: This study showed that most health care providers fail in their explanation of some essential steps in the correct use of inhalation devices.

Keywords: Inhalation Devices; Inhalation Technique; Azores Archipelago. 


\author{
ANEXO I \\ QUESTIONÁRIO \\ Avaliação do conhecimento sobre a utilização de inaladores entre médicos \\ e profissionais de farmácia dos Açores \\ Sofia Correia, Fábio Luz, Vanessa Amaral, Adelino Dias, Telma Miragaia
}

\section{Avaliação do conhecimento sobre a utilização de inaladores entre médicos e profissionais de farmácia dos Açores}

Questionário n. ${ }^{\circ}$

1. Idade anos

2. Género
$\square$ Masculino
$\square$ Feminino

3. Profissão

$\square$ Médico

$\square$ Farmacêutico

4. Local de Trabalho/ /Serviço

5. Anos de serviço como profissional de saúde

6. Quando prescreve ou fornece estes dispositivos costuma explicar como funcionam?
$\square$ Sempre
$\square$ Quase sempre
$\square$ Às vezes
Nem sempre
$\square$ Nunca

7. Quem deve explicar o modo de funcionamento?

$\square$ Médicos

Farmacêuticos

$\square$ Enfermeiros

Outros

\section{Administração de Inalador Pressurizado}

1. $\square$ Agitar o inalador doseável antes de o usar.

2. $\square$ Segurar o aerossol com a base da cápsula de metal voltada para cima, entre os dedos com o polegar sobre a base.

3. $\square$ Esvaziar os pulmões tanto quanto possível (expiração máxima).

4. $\square$ Ajustar a boca firmemente em torno do bucal.

5. $\square$ Inspirar pela boca progressivamente e comprimir o inalador.

6. $\square$ Suster a respiração pelo menos 5 segundos.

7. $\square$ Lavar a boca com água se o inalador contiver corticóides.

\section{Administração de Inalador em Pó Turbohaler}

1. $\square$ Rodar a base nos 2 sentidos até se ouvir um "click".

2. $\square$ Esvaziar os pulmões tanto quanto possível (expiração máxima).

3. $\square$ Ajustar a boca firmemente em torno do bucal.

4. $\square$ Inspiração pela boca rápida e vigorosa.

5. $\square$ Suster a respiração pelo menos 5 segundos.

6. $\square$ Lavar a boca com água se o inalador contiver corticóides. 